\title{
Wisteria floribunda agglutinin-binding glycan expression is decreased in endometriomata
}

\author{
Tomoko Hirakawa ${ }^{1}$, Kaei Nasu ${ }^{1,2^{*}}$, Kentaro Kai ${ }^{1}$, Yoko Aoyagi ${ }^{1}$, Terukazu Ishii ${ }^{1}$, Tetsuya Uemura ${ }^{1}$, \\ Mitsutake Yano ${ }^{1}$ and Hisashi Narahara ${ }^{1}$
}

\begin{abstract}
Background: Glycosylation is one of the most common post-translational modifications of eukaryotic proteins and is known to undergo dynamic changes in a wide range of biological processes. To date, however, the glycan expression profiles in endometriosis are largely unknown. The objective of the study was to identify the panel of glycans that were aberrantly expressed in endometriosis, a hormone-dependent disease.

Methods: The glycan expression profiles in primary cultured human endometriotic cyst stromal cells (ECSCs) and normal endometrial stromal cells (NESCs) were determined by lectin microarray analysis. Distribution of Wisteria floribunda agglutinin (WFA)-binding glycans in ovarian endometriotic cysts and eutopic proliferative phase endometrium were assessed by lectin histochemistry. The expressions of $\mathrm{N}$-acetylgalactosaminyl transferases that synthesize WFA-binding glycans were evaluated in ECSCs and NESCs.

Results: We found that the levels of WFA-binding glycans were decreased in ECSCs. Lectin histochemistry revealed that WFA-binding glycans were decreased only in the stromal components of the ovarian endometriotic cysts, but not in the epithelial components, compared to the eutopic proliferative phase endometrium. The expressions of $\mathrm{N}$-acetylgalactosaminyl transferases that synthesize WFA-binding glycans were downregulated in ECSCS.

Conclusions: Utilizing lectin microarray analysis and lectin histochemistry, we found that WFA-binding glycans were decreased in endometriosis. The synthetic enzymes of WFA-binding glycans were significantly downregulated in ECSCs. It is suggested that reduced expression of N-glycans with WFA-binding properties on ECSCs is a novel characteristics of endometriosis.
\end{abstract}

Keywords: Endometriosis, Wisteria floribunda agglutinin, Lectin microarray, N-acetylgalactosaminyl transferase

\section{Background}

Endometriosis is characterized by dysmenorrhea, chronic pelvic pain and/or subfertility, all of which can significantly impair the quality of life of young women, who comprise the population most likely to suffer from this estrogendependent disease [1]. Although endometriotic tissues and normal proliferative endometrium are histologically similar, several molecular differences between normal and endometriotic tissues have been identified [2-5].

In the field of reproductive medicine and oncology, glycan profiling is attracting attention because it can provide

\footnotetext{
* Correspondence: nasu@oita-u.ac.jp

'Department of Obstetrics and Gynecology, Faculty of Medicine, Oita University, Idaigaoka 1-1, Hasama-machi, Yufu-shi, Oita 879-5593, Japan ${ }^{2}$ Division of Obstetrics and Gynecology, Support System for Community Medicine, Faculty of Medicine, Oita University, Idaigaoka 1-1, Hasama-machi, Yufu-shi, Oita 879-5593, Japan
}

valuable new information about cells and tissues. It has been estimated that $1 \%$ of the human genome is involved in the production and modification of glycans [6]. Glycans are produced in a non-template-driven manner in different monosaccharides combine in a branched fashion and a variety of orientations, producing isomeric and isobaric glycans. Glycans have been shown to reflect cellular conditions during development, differentiation, adhesion, proliferation, aging, and activation, and, inflammation, infection, and tumorigenesis through specific binding to their glycan ligands [7-11]. It has also been estimated that as many as $70 \%$ of all human proteins are glycosylated [12]. As one of the most common posttranslational modifications of eukaryotic proteins, glycosylation undergoes dynamic changes in a wide range 
of biological processes [13]. However, glycan profiling in endometriosis have not been elucidated yet, which encouraged us to carry out the present glycomic study.

Glycans are bound specifically by glycan-binding proteins (GBPs), or lectins, which are diverse families of ubiquitous proteins found in viruses, fungi, bacteria, plants, and animals (including humans) [14,15]. The endogenous functions of GBPs/lectins have not been well established. Lectin microarrays, an emerging modern technology, allow the ultrasensitive detection of multiplex lectin-glycan interactions. These arrays have been used extensively in studies of pluripotent and somatic stem cells and carcinogenesis [16-23].

The present study was designed to identify the panel of glycans that were aberrantly expressed in primary cultured human endometriotic cyst stromal cells (ECSCs) in comparison with primary cultured human normal endometrial stromal cells (NESCs). Lectin microarray analysis revealed Wisteria floribunda agglutinin (WFA)binding glycans were significantly decreased in ECSCs compared to NESCs. Thereafter, we focused on WFA and evaluated the distribution of WFA-binding glycans in the ovarian endometriotic cysts and to the eutopic proliferative phase endometrium by lectin histochemistry. The expressions of $\mathrm{N}$-acetylgalactosaminyl transferases that synthesize WFA-binding glycans were also examined in ECSCs and NESCs. The present study may provide a platform for the future studies on aberrant glycan expressions in endometriosis.

\section{Methods}

\section{Ethical approval}

The present study was approved by the institutional review board of the Faculty of Medicine, Oita University, and signed informed consent was obtained from all patients (Reference No.: P-10-04; Date of approval: July 23, 2010). This study was conducted between September 1, 2013 and May 13, 2014.

\section{Tissue collection, ECSC and endometrial stromal cell (NESC) isolation procedure}

Endometriotic tissues were obtained from premenopausal patients who had undergone a surgery for ovarian endometriotic cysts ( $\mathrm{n}=35$ in total, aged 26-37 years). Normal endometrial tissues were obtained from premenopausal patients who had undergone hysterectomies for subserosal leiomyoma and had no evidence of endometriosis ( $n=39$ in total, aged 35-41 years). None of the patients had received any hormonal treatments for at least 2 years prior to the operation. All of the specimens were diagnosed as being in the mid- to late-proliferative phases, using a standard histological examination of the endometrial tissues.
Of these collected samples, sixteen endometriotic tissues and twenty normal endometrial tissues were fixed in $7 \%$ neutral buffered formaldehyde solution, embedded in paraffin, and processed for lectin histochemical staining [24].

Whereas, other 19 ovarian endometriotic tissues and 19 normal eutopic endometrial tissues were processed for cell isolation, as previously described [25,26]. Briefly, the tissues were minced in Hank's balanced salt solution and digested with $0.5 \%$ collagenase (Gibco-BRL, Gaithersburg, MD, USA) in Dulbecco's modified Eagle's medium (DMEM) (Gibco-BRL) at $37^{\circ} \mathrm{C}$ for $40 \mathrm{~min}$. The dispersed cells were filtered through a $70-\mu \mathrm{m}$ nylon mesh to remove the undigested tissue pieces. The filtrated fraction was further separated from epithelial cell clumps by differential sedimentation at unit gravity as follows. The cells were resuspended in $2 \mathrm{ml}$ of culture medium and layered slowly over $10 \mathrm{ml}$ of the medium in a centrifuge tube. Sealed tubes were placed in an upright position at $37^{\circ} \mathrm{C}$ in $5 \% \mathrm{CO}_{2}$ in air for $30 \mathrm{~min}$. After sedimentation, the top $8 \mathrm{ml}$ of the medium was collected. Finally, the medium containing stromal cells was filtered through a $40-\mu$ m nylon mesh.

Final purification was achieved by allowing stromal cells, which rapidly attached to plates, to adhere selectively to culture dishes for $30 \mathrm{~min}$ at $37^{\circ} \mathrm{C}$, followed by the removal of non-adhering epithelial cells. Isolated ECSCs and NESCs were cultured in DMEM supplemented with $100 \mathrm{IU} / \mathrm{ml}$ of penicillin (Gibco-BRL), $50 \mathrm{mg} / \mathrm{ml}$ of streptomycin (Gibco-BRL) and 10\% heatinactivated fetal bovine serum (FBS) (Gibco-BRL) at $37^{\circ} \mathrm{C}$ in $5 \% \mathrm{CO} 2$ in air. ECSCs and NESCs in monolayer cultures after the third passage were $>99 \%$ pure as analysed by immunocytochemical staining with antibodies against vimentin (V9; Dako, Copenhagen, Denmark), CD10 (SS2/36; Dako), cytokeratin (Dako), factor VIII (Dako) and leukocyte common antigen (2B11 t PD7/26, Dako) and were used for the following experiments $[25,26]$. The cells separately isolated from individual patients were used in each experiment.

\section{Protein extraction for lectin microarray}

Cultured ECSCs $(n=9)$ and NESCs $(n=11)$ were harvested by trypsinization. The cells were pelleted by washing with phosphate-buffered saline (PBS) by centrifugation, then solubilized with $20 \mu \mathrm{l}$ of PBS containing 0.5\% Nonidet P40, and sonicated gently. The cell suspensions were incubated on ice for 60 minutes and centrifuged at $20,000 \mathrm{~g}$ for $5 \mathrm{~min}$ at $4^{\circ} \mathrm{C}$. The obtained supernatants were used as detergent-solubilized glycoprotein extract.

\section{Lectin microarray analysis}

Lectin microarray analysis was performed according to the manufacturer's instruction. Briefly, each $20 \mu \mathrm{l}$ aliquot of the above glycoprotein solutions was incubated with 
$10 \mu \mathrm{g}$ of Cy3-succimidyl ester (Amersham Biosciences, Tokyo, Japan) for 60 minutes at room temperature in the dark. The obtained Cy3-labeled glycoprotein was subjected to the lectin chip (LecChipTM; GP Biosciences, Yokohama, Japan) that contain 45 lectins for human cells. One hundred microliters of Cy3-labeled glycoprotein solution in probing buffer (Tris-buffered saline containing 0.05\% Triton X-100) were applied to each well with immobilized lectins, at a concentration of $125 \mathrm{ng} / \mathrm{ml}$. Incubation was performed at $4^{\circ} \mathrm{C}$ for about 15 hours until the binding reached equilibrium. After the incubation, we acquired a fluorescence image of the array using an evanescent-field fluorescence scanner, GlycoStation ${ }^{\text {TM }}$ Reader 1200, SC-Profiler (GP BioScience). We calculated the net intensity value for each spot by subtracting a background value from signal intensity and averaged the signal net intensity values of three spots. To calculate the fold change of each lectin-binding glycans in ECSCs $(n=9)$ relative to NESCs $(n=11)$, the data for a given lectin were normalized to the median of that lectin in the eleven NESCs. Among 45 lectins, differentially expressed lectins were statistically extracted by a fold change of $>2.0 \mathrm{com}-$ bined with two-sided Mann-Whitney $U$ test, with the Benjamini-Hochberg adjustment for false discovery rate (FDR) $(p<0.05)$.

\section{Lectin histochemistry}

Lectin histochemical staining using streptavidin-biotinperoxidase method was performed with biotinylated WFA (B-1355, Vector Laboratories, Peterborough, UK). Dewaxed and hydrated sections of ovarian endometriotic cyst $(n=16)$ and proliferative phase eutopic endometrium $(\mathrm{n}=20)$ were immersed in $10 \mathrm{mM}$ citric acid $\left(\mathrm{pH}\right.$ 6.0) and autoclaved at $120^{\circ} \mathrm{C}$ for $10 \mathrm{~min}$. Antigen activated sections were immersed in $0.3 \%$ hydrogen peroxide in methanol at room temperature for $10 \mathrm{~min}$ to block endogenous peroxidase activity, rinsed, microwaved in ethylenediaminetetraacetic acid buffer $(1 \mathrm{mM}$, $\mathrm{pH}$ 8.0) for $10 \mathrm{~min}$ and equilibrated in PBS. Sections were blocked with $1 \%$ bovine serum albumin at room temperature for $10 \mathrm{~min}$, before overnight incubation in $10 \mu \mathrm{g} / \mathrm{ml}$ biotinylated WFA at room temperature for $150 \mathrm{~min}$. The sections were rinsed in PBS before incubation with Streptavidin-peroxidase reagent (424021, Histofine simple stain SAB-PO (M) kit, Nichirei Co., Tokyo, Japan) at room temperature for $5 \mathrm{~min}$. Subsequently, the sections were rinsed in distilled water before 60 sec-incubation with 3,3'-diaminobenzidine tetra hydrochloride (Histofine DAB substrate kit, Nichirei Co.). Sections were washed in distilled water, counterstained with hematoxylin, dehydrated, cleared and mounted. Histochemical staining score was used to assess the staining intensity with light microscopy (Zeiss Axiophot; Carl Zeiss, Oberkochen, Germany).
Total RNA isolation and quantitative reverse transcription-polymerase chain reaction (RT-PCR) for $\mathrm{N}$-acetylgalactosaminyl transferases

WFA binds with high affinity to glycans containing $\mathrm{N}$ acetyl-D-galactosamine (GalNAc), namely GalNAcb13Gal, GalNAcb1-4Gal, and GalNAcb1-4GlcNAc [27]. Whereas, the cognate glycosyltransferases of WFA include $\beta 1,3-\mathrm{N}$-acetylgalactosaminyl transferase (B3GALNT) 1, B3GALNT2, $\beta 1,4-\mathrm{N}$-acetylgalactosaminyltransferase (B4 GALNT) 1, B4GALNT2, B4GALNT3, and B4GALNT4 [28]. Therefore, we next evaluated the expression of these $\mathrm{N}$-acetylgalactosaminyl transferases in ECSCs and NESCs.

Total RNA from cultured ECSCs $(n=7)$ and NESCs $(\mathrm{n}=5)$ was extracted with a RNeasy Mini kit (Qiagen, Valencia, CA, USA). The quality of the extracted RNA was confirmed by measuring the absorbance at $230 \mathrm{~nm}$, $260 \mathrm{~nm}$, and $280 \mathrm{~nm}$ using a spectrophotometer (NanoDrop 2000, Thermo Scientific, Wilmington, DE, USA), and subjected to RT-PCR as described previously [24].

Quantitative RT-PCR was carried out in with a LightCycler 480 (Roche Diagnostics, Penzberg, Germany) using the TaqMan Universal PCR Master Mix II No AmpErase UNG (Applied Biosystems) with B3GALNT1specific (Assay ID: Hs00364202_s1, Applied Biosystems, Carlsbad, CA, USA), B3GALNT2-specific (Assay ID: Hs00 380823_m1, Applied Biosystems), B4GALNT1-specific (Assay ID: Hs00155195_m1, Applied Biosystems), B4G ALNT2-specific (Assay ID: Hs00396440_m1, Applied Biosystems), B4GALNT3-specific (Assay ID: Hs0041 9636_m1, Applied Biosystems), B4GALNT4-specific (Assay ID: Hs00331790_m1, Applied Biosystems) or glyceraldehyde 3-phosphate dehydrogenase (GAPDH)-specific (Assay ID: Hs02758991_g1, Applied Biosystems) primers [24]. Data from the RT-PCR experiments are presented as the percentage of each $\mathrm{N}$-acetylgalactosaminyl transferase/ GAPDH of ECSCs to those of NESCs.

\section{Western blot analysis}

The protein levels of $\mathrm{N}$-acetylgalactosaminyl transferases in ECSCs $(n=3)$ and NESCs $(n=3)$ were investigated by Western blot analysis [24]. Antibodies against B3GALNT1 (ab98873, Abcam, Tokyo, Japan), B3GALNT2 (ab156785, Abcam), B4GALNT1 (ab55065, Abcam), B4GALNT2 (ab176856, Abcam), B4GALNT3 (ab82800, Abcam), B4GALNT4 (sc-166982, Santa Cruz Biotechnology, CA, USA) and GAPDH (Ambion, Austin, TX, USA) were used as primary antibodies. The relative expression of each $\mathrm{N}$ acetylgalactosaminyl transferase protein in ECSCs and NESCs was analyzed using Image Lab $^{\text {TM }}$ software (Bio-Rad Laboratories, Hercules, CA, USA).

\section{Statistical analysis}

Data were calculated as percentages to the corresponding controls, presented as means $\pm \mathrm{SD}$, and were appropriately 
analyzed by the two-sided Mann-Whitney $U$ test, the Student's $t$ test, or Bonferroni test with Sigmaplot 11.2 (Systat Software, San Jose, CA, USA). Values of $p<0.05$ were considered statistically significant.

\section{Results}

Identification of glycans differentially expressed in ECSCs and NESCs

We used a lectin microarray platform covering a total of 45 human lectins to determine the expression patterns of glycans in ECSCs $(n=9)$ and NESCs $(n=11)$. Unsupervised hierarchical cluster analysis of these 45 lectins showed that the patterns of glycan expression in ECSCs were similar to those in NESCs as a whole (Figure 1), suggesting that diseased tissues in endometriosis retain the characteristics of their origin, namely the eutopic endometrium, concerning lectin-binding glycan expression profiles.

To identify the group of glycans that are differentially expressed between ECSCs and NESCs, we chose lectinbinding glycans with a fold change of $>2.0$ from 45 lectins. As shown in Table 1, we found that only WFA-binding glycans were significantly downregulated in ECSCs by using the two-sided Mann-Whitney $U$ test and adjusted FDR of $<0.05$. Whereas, none of the lectin-binding glycans were found to be statistically upregulated in ECSCs compared to NESCs.

Distribution of WFA-binding glycans in ovarian endometriotic cysts and normal eutopic endometrium

We focused on WFA for the following experiments. Both the glandular epithelial cells and the stromal component of ovarian endometriotic cysts as well as the normal proliferative phase endometrium were positive for WFAbinding glycans with strong to weak intensity (Figure 2 and Table 2). Significantly decreased staining for WFAbinding glycans was observed in endometriotic stromal component in comparison with eutopic endometrium. Whereas, similar staining patterns were observed in the epithelial component in these tissues.

\section{Messenger RNA (mRNA) expression of $\mathrm{N}$-acetylgalactosaminyl transferases in ECSCs and NESCs}

The mRNA expressions of B3GALNT1, B3GALNT2, B4GALNT1, B4GALNT2, B4GALNT3, and B4GALNT4 in ECSCs and NESCs were evaluated by quantitative RTPCR. As shown in Figure 3, the mRNA expressions of B3GALNT2, B4GALNT1, B4GALNT2, B4GALNT3, and B4GALNT4 in ECSCs were significantly lower than those in NESCs. Although there was no significance, the mRNA expression of B3GALNT1 tends to decrease in ECSCs compared to NESCs.

\section{Protein levels of $\mathrm{N}$-acetylgalactosaminyl transferases in ECSCs and NESCs}

The protein expressions of B3GALNT1, B3GALNT2, B4GALNT1, B4GALNT2, B4GALNT3, and B4GALNT4 in ECSCs and NESCs were evaluated by Western blot analysis. As shown in Figure 4, the protein expressions of B3GALNT1, B3GALNT2, B4GALNT2, and B4GALNT3 were detected in ECSCs and NESCs. The levels of these four $\mathrm{N}$-acetylgalactosaminyl transferases were significant higher in NESCs compared to ECSCs. Whereas, the protein

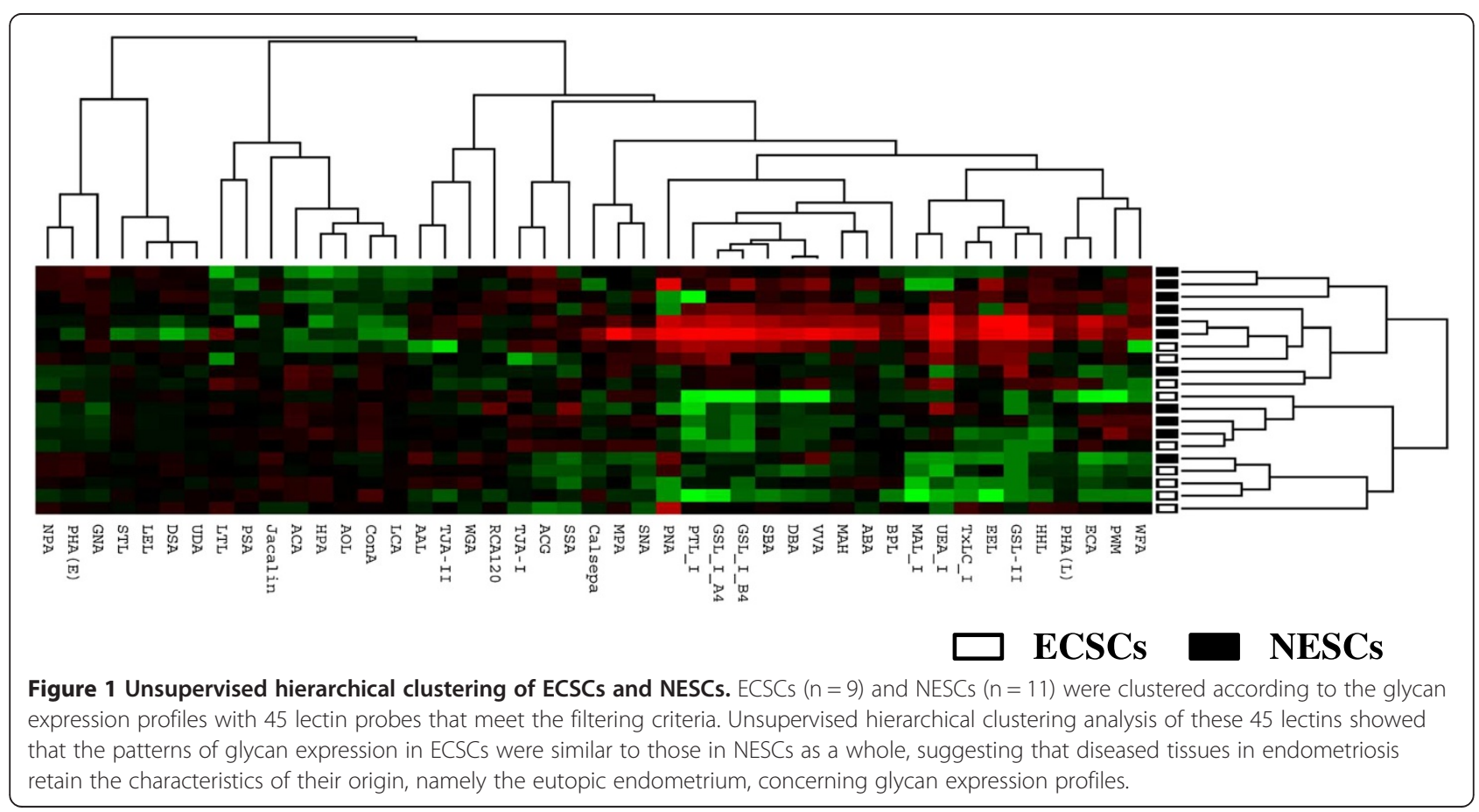


Table 1 The lectin signals between ECSCs $(n=9)$ and NESCs $(n=11)$ obtained from a lectin microarray analysis

\begin{tabular}{|c|c|c|c|c|}
\hline Lectin & $\begin{array}{l}\text { ECSC signals } \\
(\text { Mean } \pm \text { SD) }\end{array}$ & $\begin{array}{l}\text { NESC signals } \\
\text { (Mean } \pm \text { SD) }\end{array}$ & $\begin{array}{l}\text { ECSC/NESC } \\
\text { Signal ratio }\end{array}$ & $\begin{array}{l}\text { FDR-adjusted } \\
p \text {-value }\end{array}$ \\
\hline$\overline{G S L-I I}$ & $2.9111 \pm 2.685$ & $12.773 \pm 21.907$ & 0.228 & 0.506 \\
\hline WFA & $8.578 \pm 4.499$ & $23.691 \pm 10.474$ & 0.362 & 0.031 \\
\hline EEL & $10.422 \pm 7.788$ & $24.227 \pm 33.06$ & 0.430 & 0.442 \\
\hline GSL_I_A4 & $14.5 \pm 14.009$ & $33.018 \pm 51.382$ & 0.439 & 0.459 \\
\hline GSL_I_B4 & $12.344 \pm 12.107$ & $27.973 \pm 33.193$ & 0.441 & 0.481 \\
\hline WA & $11.122 \pm 7.198$ & $24.918 \pm 22.587$ & 0.446 & 0.596 \\
\hline ECA & $9.722 \pm 5.908$ & $19.2 \pm 17.569$ & 0.506 & 0.59 \\
\hline UEA_I & $6.033 \pm 5.96$ & $11.909 \pm 14.76$ & 0.507 & 0.486 \\
\hline DBA & $12.133 \pm 8.693$ & $23.536 \pm 21.247$ & 0.516 & 0.521 \\
\hline PTL_I & $12.2 \pm 12.806$ & $22.136 \pm 33.735$ & 0.551 & 0.508 \\
\hline SBA & $13.178 \pm 10.764$ & $23.7 \pm 20.775$ & 0.556 & 0.571 \\
\hline $\mathrm{HHL}$ & $17 \pm 9.687$ & $30.473 \pm 28.645$ & 0.558 & 0.541 \\
\hline PWM & $9.744 \pm 5.585$ & $17.182 \pm 9.881$ & 0.567 & 0.45 \\
\hline $\mathrm{MAH}$ & $16.8 \pm 5.232$ & $28.836 \pm 27.646$ & 0.583 & 0.464 \\
\hline ABA & $90.233 \pm 22.797$ & $143.064 \pm 120.478$ & 0.631 & 0.483 \\
\hline PNA & $4.967 \pm 4.438$ & $7.791 \pm 8.819$ & 0.637 & 0.501 \\
\hline MPA & $15.444 \pm 4.319$ & $22.818 \pm 30.326$ & 0.677 & 0.554 \\
\hline TXLC_I & $17.422 \pm 9.327$ & $24.845 \pm 19.056$ & 0.701 & 0.492 \\
\hline ACG & $64.922 \pm 34.0556$ & $92.445 \pm 37.676$ & 0.702 & 0.585 \\
\hline MAL_I & $18.056 \pm 12.372$ & $24.864 \pm 22.1$ & 0.726 & 0.511 \\
\hline $\mathrm{BPL}$ & $25.8 \pm 7.434$ & $34.618 \pm 15.621$ & 0.745 & 0.533 \\
\hline SNA & $33.144 \pm 13.886$ & $44.355 \pm 34.96$ & 0.747 & 0.488 \\
\hline SSA & $36.811 \pm 15.962$ & $48.909 \pm 27.953$ & 0.753 & 0.495 \\
\hline $\mathrm{PHA}(\mathrm{L})$ & $12.533 \pm 5.879$ & $16.264 \pm 6.687$ & 0.771 & 0.453 \\
\hline RCA120 & $66.022 \pm 16.372$ & $82.918 \pm 31.243$ & 0.796 & 0.528 \\
\hline TJA-II & $37.367 \pm 15.26$ & $45.945 \pm 12.496$ & 0.813 & 0.461 \\
\hline GNA & $93.978 \pm 23.352$ & $109.018 \pm 43.165$ & 0.862 & 0.504 \\
\hline WGA & $98.011 \pm 25.708$ & $112.455 \pm 31.601$ & 0.872 & 0.475 \\
\hline TJA-I & $49.278 \pm 24.915$ & $55.745 \pm 16.598$ & 0.884 & 0.611 \\
\hline AAL & $52.178 \pm 18.876$ & $58.445 \pm 20.733$ & 0.893 & 0.595 \\
\hline Calsepa & $45.089 \pm 15.359$ & $49.445 \pm 28.858$ & 0.912 & 0.775 \\
\hline Jacalin & $168.8 \pm 35.876$ & $172.218 \pm 42.136$ & 0.98 & 0.847 \\
\hline STL & $679.567 \pm 70.057$ & $656.364 \pm 168.174$ & 1.0354 & 0.77 \\
\hline NPA & $179.722 \pm 54.043$ & $172.7 \pm 50.535$ & 1.0407 & 0.787 \\
\hline $\mathrm{PHA}(\mathrm{E})$ & $146.678 \pm 36.955$ & $139.018 \pm 49.862$ & 1.055 & 0.766 \\
\hline ACA & $145.689 \pm 51.242$ & $136.455 \pm 66.262$ & 1.068 & 0.763 \\
\hline LTL & $33.667 \pm 13.814$ & $31.255 \pm 16.35$ & 1.077 & 0.777 \\
\hline LEL & $548 \pm 50.485$ & $508.155 \pm 117.768$ & 1.078 & 0.508 \\
\hline UDA & $313.967 \pm 37.292$ & $285.464 \pm 71.134$ & 1.1 & 0.502 \\
\hline DSA & $443.7 \pm 77.582$ & $396.864 \pm 132.835$ & 1.118 & 0.494 \\
\hline HPA & $89.722 \pm 26.453$ & $73.982 \pm 41.287$ & 1.213 & 0.509 \\
\hline PSA & $85.311 \pm 14.361$ & $66.655 \pm 22.578$ & 1.28 & 0.433 \\
\hline $\mathrm{AOL}$ & $91.611 \pm 17.239$ & $70.855 \pm 24.037$ & 1.293 & 0.568 \\
\hline LCA & $263.311 \pm 42.57$ & $198.2 \pm 70.68$ & 1.329 & 0.477 \\
\hline ConA & $393.767 \pm 112.746$ & $294.991 \pm 141.82$ & 1.335 & 0.642 \\
\hline
\end{tabular}

FDR, false discovery rate. 


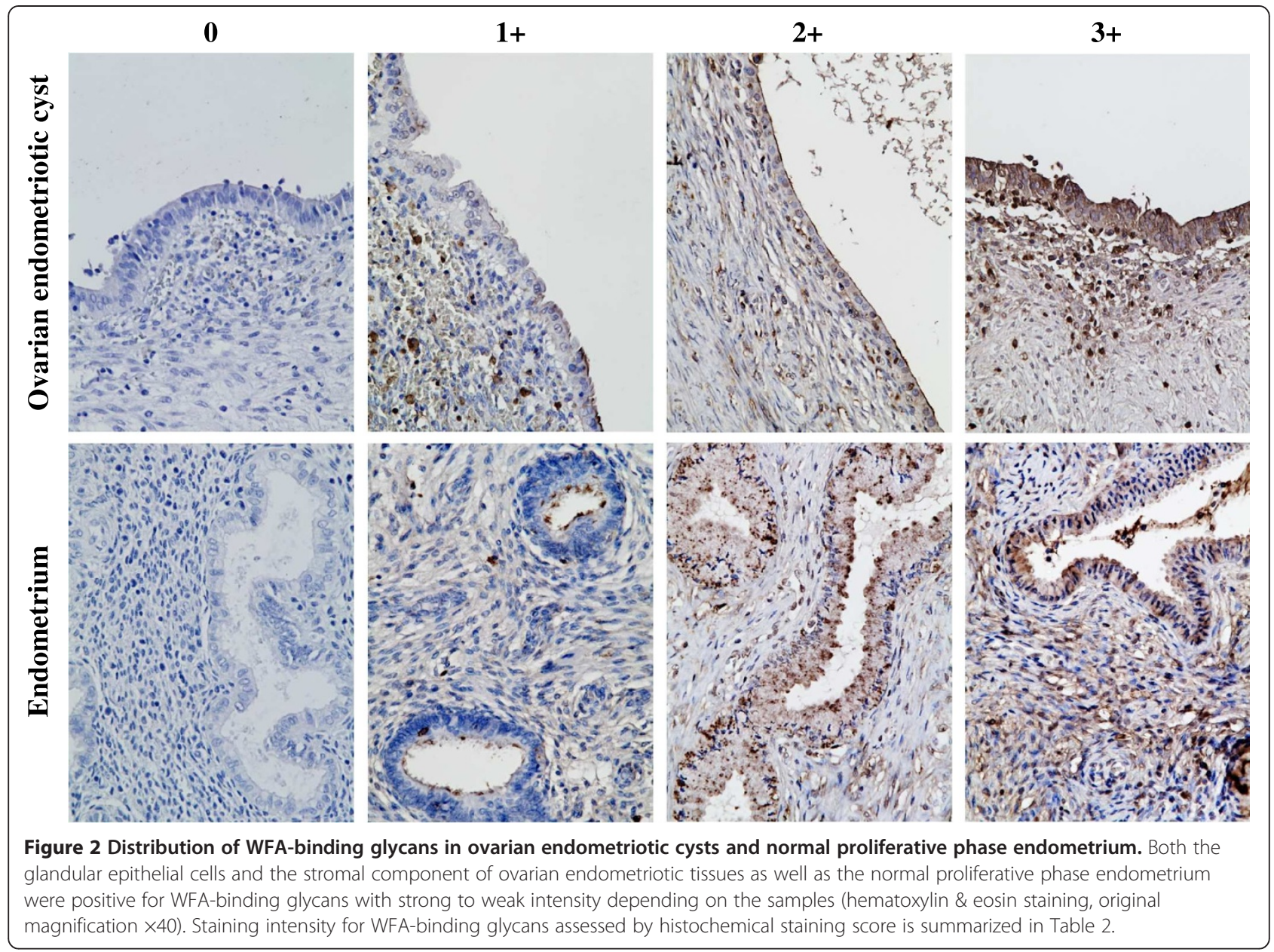

expressions of B4GALNT1 and B4GALNT4 in ECSCs and NESCs were under the detection levels.

\section{Discussion}

The current study was conducted to identify the panel of aberrantly expressed glycans in ECSCs in comparison with those in NESCs. Utilizing the lectin microarray technique, we found, for the first time, that WFA-binding glycans were decreased in ECSCs. Lectin histochemistry revealed that WFA-binding glycans were decreased only in the stromal components of the ovarian endometriotic cysts, but not in the epithelial components, compared to the eutopic proliferative phase endometrium. Thereafter, we focused on the synthetic enzymes of WFA-binding glycans and designed further experiments. The mRNA levels of B3GALNT2, B4GALNT1, B4GALNT2, B4GALNT3, and B4GALNT4 were significantly downregulated in ECSCs, whereas the protein levels of B3GALNT1, B3GALNT2,

Table 2 WFA-staining scores in ovarian endometriotic cysts $(n=16)$ and proliferative-phase eutopic endometrium samples $(n=20)$

\begin{tabular}{|c|c|c|c|c|}
\hline \multirow[b]{2}{*}{ WFA-staining score } & \multicolumn{2}{|c|}{ Epithelial component } & \multicolumn{2}{|c|}{ Stromal component } \\
\hline & Endometriotic cyst & Eutopic endometrium & Endometriotic cyst & Eutopic endometrium \\
\hline 3 & 7 & 12 & 3 & 9 \\
\hline 2 & 6 & 5 & 5 & 7 \\
\hline 1 & 3 & 3 & 7 & 4 \\
\hline 0 & 0 & 0 & 1 & 0 \\
\hline Mean \pm SD & $2.3 \pm 0.8$ & $2.5 \pm 0.8$ & $1.6 \pm 0.9^{*}$ & $2.3 \pm 0.8$ \\
\hline
\end{tabular}

The WFA-staining intensity was evaluated separately for the epithelial component and the stromal component and stratified as follows: 0 (negative), 1 (weak), 2 (moderate), and 3 (strong). Two gynecologists (K. K. and M. Y.) scored all samples independently, and discrepancies were resolved by simultaneous examination. ${ }^{*} p<0.05$ vs. eutopic endometrium (Student's $t$-test). 


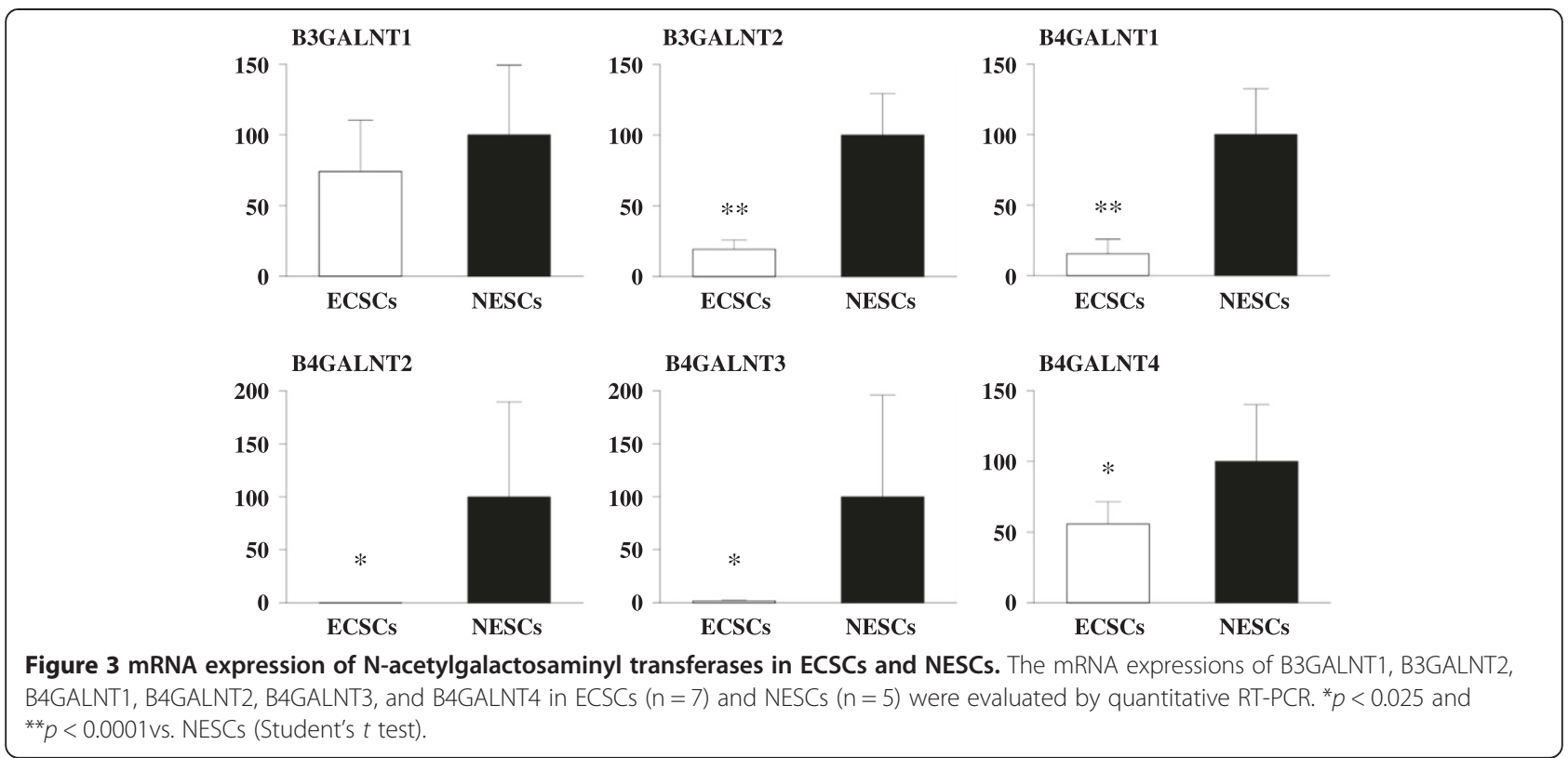

B4GALNT2, and B4GALNT3 were significantly downregulated in ECSCs. The meanings of aberrant glycan expressions in endometriosis are not elucidated from the present study, however, it is speculated that aberrantly expressed glycans in ECSCs may involve in the development of disease-specific features of endometriosis. The present findings may provide a platform for the future glycomic studies in endometriosis.

A huge number of possible unique sequences can be composed of glycans, from basic building units. Glycans are thus excellent information-coding "tools". Cummings [29] estimated that up to 500,000 glycan-modified biomolecules may form a cellular glycome, based on 7,000 unique glycan sequences. Arnaud et al. [30] estimated that in humans, $70 \%$ of proteins and $(80 \%$ of membrane-bound proteins) are glycosylated. Indeed, glycosylation is a very common type of post-translational modification of proteins, with important roles in a wide range of cellular events. Cell development, differentiation, morphogenesis, proliferation, adhesion, fertilization, embryogenesis, immunity, infection, and tumorigenesis have all been shown to involve protein glycosylation [7-11,31-33].

Oncology researchers have studied aberrant glycosylation extensively. For example, the invasion and metastatic potential of cancer cells were found to be strongly correlated cell surface sialylation and the $\beta 1-6$ branching of $\mathrm{N}$-glycans [33-36]. The heavily O-glycosylated tumorassociated mucin known as sialylated mucin 1 [37] expresses Tn (GalNAc) antigens and T (Galb1, 3GalNAc) antigens that are linked to cellular adhesion and functioning of the immune system [38,39]. In addition, several glycan-based biomarkers have been established, including $\alpha$-fetoprotein 1 [40], prostate-specific antigen [41], the carcino-embryonic antigen family [42,43], and cancer antigen 19-9 [44]. The complete array of glycans associated with endometriosis has not been determined.

Lectins each have a unique specificity profile, and the differing specificities for complex glycans make lectins a valuable tool for identifying a particular type of glycan. Together the lectins comprise a diverse group of carbohydrate-binding proteins [10]. Lectin microarrays are used to profile the many types of glycan structures observed at the cell surface and in glycoconjugates [10]. Berkes et al. [45] evaluated the plasma N-glycan levels in endometriosis patients by hydrophilic interaction high performance liquid chromatography and found the significant decrease of some of the glycan peaks. In the present study, we used a recently developed lectin microarray that consists of 45 lectins with different binding preferences covering $\mathrm{N}$ - and $\mathrm{O}$-linked glycans [23] and we found that WFA-binding glycans were decreased in ECSCs.

The reactivity of WFA is well-documented in mammary glands [46], cholangiocarcinoma [16,17], prostate cancer [47], and the central nervous system [48]. WFA reactivity was shown to be upregulated in prostate cancer [47] and cholangiocarcinoma [17]. Matsuda et al. [17] demonstrated that WFA reactivity was associated with MUC1 in cholangiocarcinoma. WFA specifically recognizes terminal $\alpha / \beta-\mathrm{N}$ acetylgalactosamine residues on $\mathrm{N}$-glycans $[28,46,49]$.

The genes B3GALNT1, B3GALNT2, B4GALNT2, and B4GALNT3 synthesize WFA-binding glycans [28], and we found that their expressions were significantly downregulated in ECSCs, a finding that is consistent with the present lectin microarray and lectin histochemistry results. In some tumors, glycosyltransferase expression levels were shown to be up- or down-regulated [50-53]. We did not 
(A)

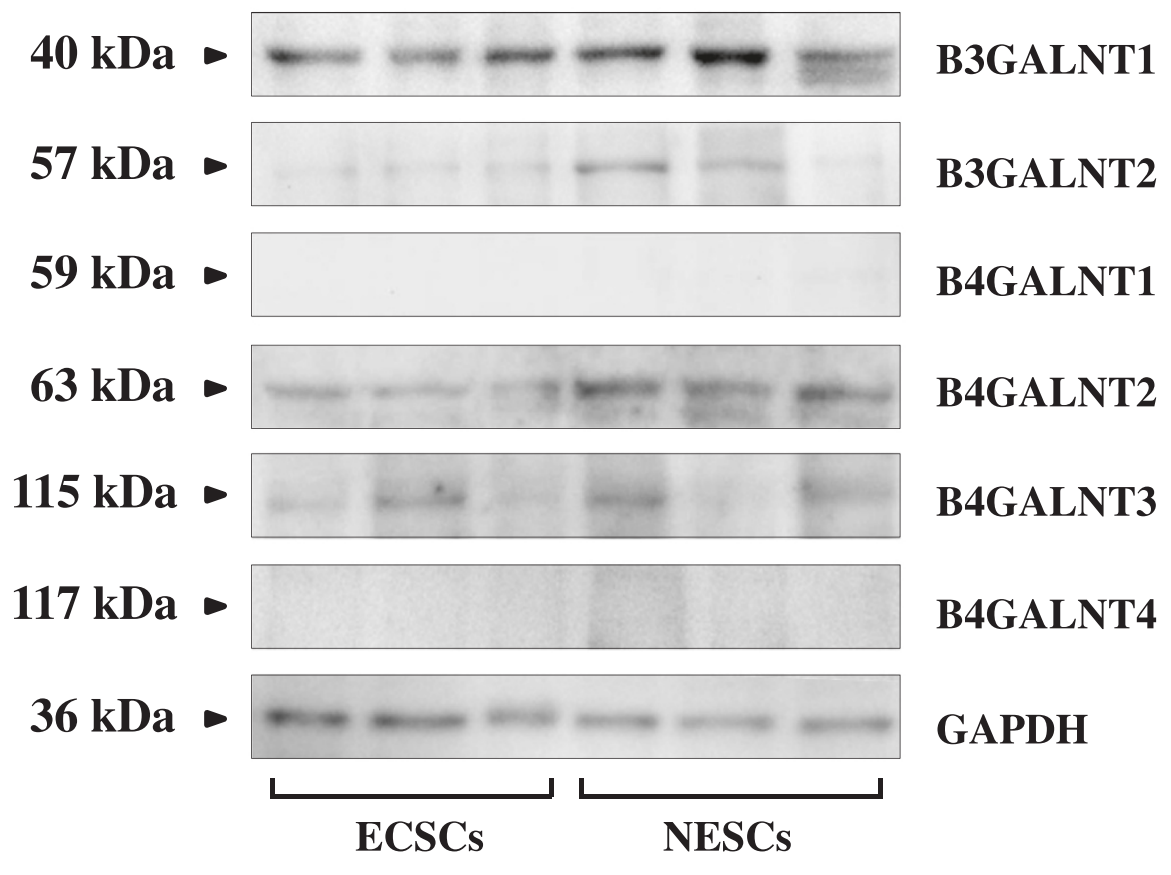

(B)
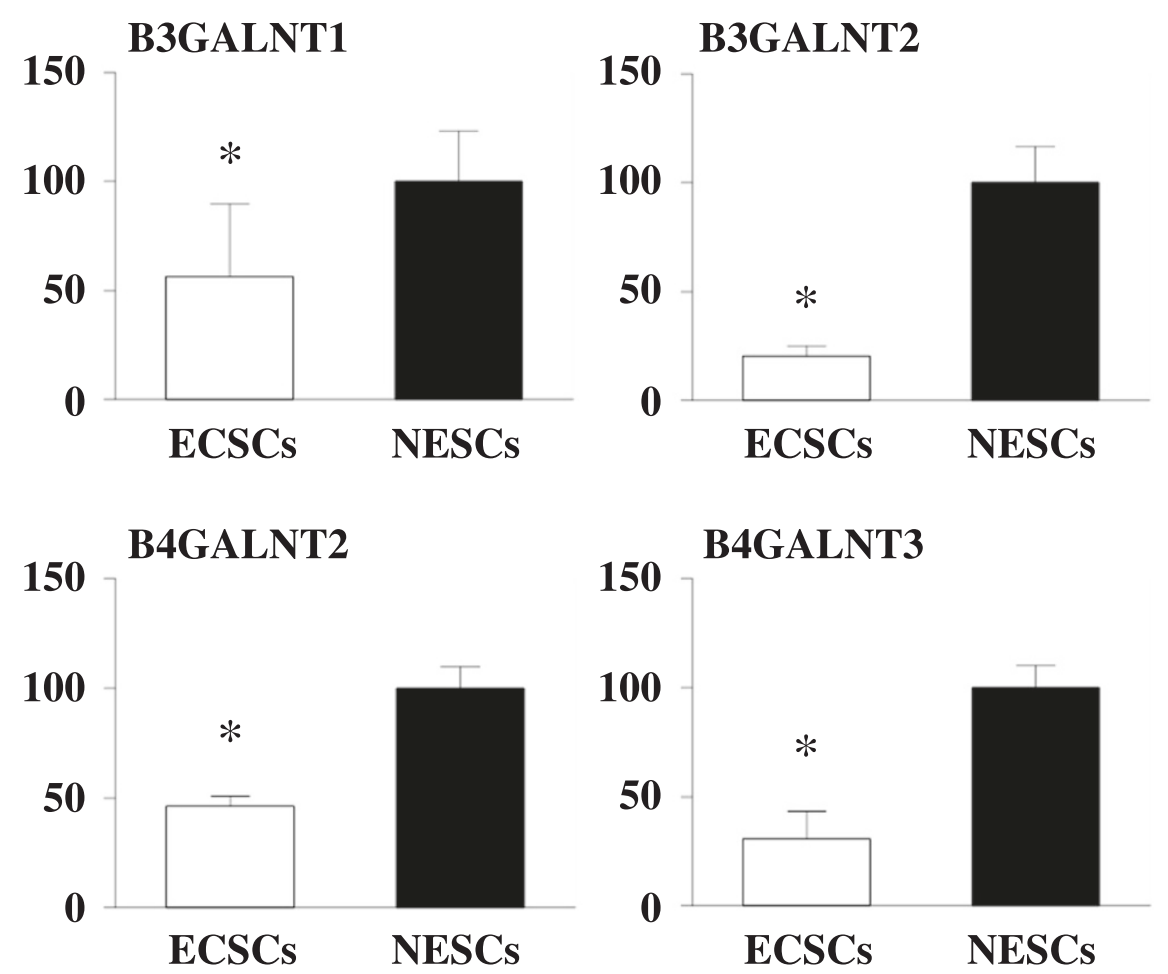

Figure 4 Protein levels of $\mathrm{N}$-acetylgalactosaminyl transferases in ECSCs and NESCs. (A) Results of Western blot analysis. (B) Relative protein levels of N-acetylgalactosaminyl transferases in ECSCs $(n=3)$ and NESCs $(n=3)$. The levels of B3GALNT1, B3GALNT2, B4GALNT2, and B4GALNT3 were significant higher in NESCs compared to ECSCs. Whereas, the protein expressions of B4GALNT1 and B4GALNT4 in ECSCS and NESCs were under the detection levels. ${ }^{*} p<0.005$ vs. NESCs (Student's $t$ test). 
evaluate which glycoproteins were affected by these decreased $\mathrm{N}$-acetylgalactosaminyl transferases in the present study. Further detailed examinations of this matter are needed to elucidate the importance of aberrant glycan expression in the pathogenesis of endometriosis.

To our knowledge, hormonal regulation of glycan expressions in the normal cyclic endometrium as well as the diseased endometrium has not been elucidated yet. Whereas, we did not evaluate the glycan profiles in the eutopic endometrium of the endometriosis patients in the present study. These are the limitations of the present study and we would like to elucidate these points in the future.

\section{Conclusions}

Utilizing lectin microarray analysis and lectin histochemistry, we found that WFA-binding glycans were decreased in ECSCs. The synthetic enzymes of WFA-binding glycans were significantly downregulated in ECSCs. It is suggested that reduced expression of $\mathrm{N}$-glycans with WFA-binding properties on ECSCs is a novel characteristics of endometriosis. Further studies on the repertoire of glycomics may provide useful information on the pathogenesis of endometriosis.

\begin{abstract}
Abbreviations
B3GALNT: $\beta 1,3-N$-acetylgalactosaminyl transferase; B4GALNT: $\beta 1$ 4-N-acetylgalactosaminyltransferase; ECSCs: Endometriotic cyst stromal cells; FDR: False discovery rate; GaINAc: N-acetyl-D-galactosamine; GAPDH: Glyceraldehyde 3-phosphate dehydrogenase; GBPs: Glycan-binding proteins; mRNA: Messenger RNA; NESCs: Normal endometrial stromal cells; PBS: Phosphate-buffered saline; RT-PCR: Reverse transcription-polymerase chain reaction; WFA: Wisteria floribunda agglutinin.
\end{abstract}

\section{Competing interests}

The authors declare that they have no competing interests.

\section{Authors' contributions}

$\mathrm{KN}$ and $\mathrm{HN}$ participated in the study design, analysis and manuscript drafting. TH, KK, YA, TI, TU, and MY executed the study. All the authors read and approved the final manuscript.

\section{Acknowledgements \\ This work was supported in part by the Grant-in-Aids for Scientific Research from the Japan Society for the Promotion of Science (no. 13237327 to K. Nasu, no. 26861335 to K. Kai, and no. 23592407 to H. Narahara). The funders had no role in study design, data collection and analysis, decision to publish, or preparation of the manuscript.}

Received: 5 August 2014 Accepted: 13 October 2014

Published: 24 October 2014

\section{References}

1. Giudice LC, Kao LC: Endometriosis. Lancet 2004, 364:1789-1799.

2. Nasu K, Nishida M, Kawano Y, Tsuno A, Abe W, Yuge A, Takai N, Narahara H: Aberrant expression of apoptosis-related molecules in endometriosis: a possible mechanism underlying the pathogenesis of endometriosis Reprod Sci 2011, 18:206-218.

3. Nasu K, Kawano Y, Tsukamoto Y, Takano M, Takai N, Li H, Furukawa Y, Abe W, Moriyama M, Narahara $\mathrm{H}$ : Aberrant DNA methylation status of endometriosis: epigenetics as the pathogenesis, biomarker and therapeutic target. J Obstet Gynaecol Res 2011, 37:683-695.
4. Abe W, Nasu K, Nakada C, Kawano Y, Moriyama M, Narahara H: miR-196b targets c-myc and $\mathrm{Bcl}-2$ expression, inhibits proliferation and induces apoptosis in endometriotic stromal cells. Hum Reprod 2013, 28:750-761.

5. Nasu K, Kawano Y, Kai K, Aoyagi Y, Abe W, Okamoto M, Narahara H: Aberrant histone modification in endometriosis. Front Biosci 2014, 19:1202-1214.

6. Lowe JB, Marth JD: A genetic approach to Mammalian glycan function. Annu Rev Biochem 2003, 72:643-691.

7. Hakomori S: Glycosylation defining cancer malignancy: new wine in an old bottle. Proc Natl Acad Sci U S A 2002, 99:10231-10233.

8. Robinson MJ, Sancho D, Slack EC, LeibundGut-Landmann S, Reis e Sousa C: Myeloid C-type lectins in innate immunity. Nat Immunol 2006, 7:1258-1265.

9. Crocker PR, Paulson JC, Varki A: Siglecs and their roles in the immune system. Nat Rev Immunol 2007, 7:255-266.

10. Hirabayashi J: Concept, strategy and realization of lectin-based glycan profiling. J Biochem 2008, 144:139-147.

11. Dall'Olio F, Malagolini N, Trinchera M, Chiricolo M: Mechanisms of cancer-associated glycosylation changes. Front Biosci 2012, 17:670-699.

12. Apweiler $R$, Hermjakob $H$, Sharon $N$ : On the frequency of protein glycosylation, as deduced from analysis of the SWISS-PROT database. Biochim Biophys Acta 1999, 1473:4-1478.

13. Opdenakker G, Rudd PM, Ponting CP, Dwek RA: Concepts and principles of glycobiology. FASEB J 1993, 7:1330-1337.

14. Sharon N, Goldstein IJ: Lectins: more than insecticides. Science 1998, 282:1049.

15. Sharon $\mathrm{N}$, Lis $\mathrm{H}$ : History of lectins: from hemagglutinins to biological recognition molecules. Glycobiology 2004, 14:53R-62R.

16. Matsuda A, Kuno A, Ishida H, Kawamoto T, Shoda J, Hirabayashi J: Development of an all-in-one technology for glycan profiling targeting formalin-embedded tissue sections. Biochem Biophys Res Commun 2008, 370:259-263.

17. Matsuda A, Kuno A, Kawamoto $T$, Matsuzaki $H$, Irimura $T$, Ikehara $Y$, Zen $Y$, Nakanuma Y, Yamamoto M, Ohkohchi N, Shoda J, Hirabayashi J, Narimatsu $\mathrm{H}$ : Wisteria floribunda agglutinin-positive mucin 1 is a sensitive biliary marker for human cholangiocarcinoma. Hepatology 2010, 52:174-182.

18. Kuno A, Matsuda A, Ikehara Y, Narimatsu H, Hirabayashi J: Differential glycan profiling by lectin microarray targeting tissue specimens. Methods Enzymol 2010, 478:165-179.

19. Narimatsu $H$, Sawaki $H$, Kuno A, Kaji H, Ito H, Ikehara Y: A strategy for discovery of cancer glyco-biomarkers in serum using newly developed technologies for glycoproteomics. FEBS J 2010, 277:95-105.

20. Fry SA, Afrough B, Lomax-Browne HJ, Timms JF, Velentzis LS, Leathem AJ: Lectin microarray profiling of metastatic breast cancers. Glycobiology 2011, 21:1060-1070

21. Tateno H, Toyota M, Saito S, Onuma Y, Ito Y, Hiemori K, Fukumura M, Matsushima A, Nakanishi M, Ohnuma K, Akutsu H, Umezawa A, Horimoto K, Hirabayashi J, Asashima M: Glycome diagnosis of human induced pluripotent stem cells using lectin microarray. J Biol Chem 2011, 286:20345-20353.

22. Toyoda M, Yamazaki-Inoue M, Itakura Y, Kuno A, Ogawa T, Yamada M, Akutsu H, Takahashi Y, Kanzaki S, Narimatsu H, Hirabayashi J, Umezawa A: Lectin microarray analysis of pluripotent and multipotent stem cells. Genes Cells 2011, 16:1-11.

23. Hirabayashi J, Yamada M, Kuno A, Tateno H: Lectin microarrays: concept, principle and applications. Chem Soc Rev 2013, 42:4443-4458.

24. Kawano Y, Nasu K, Hijiya N, Tsukamoto Y, Amada K, Abe W, Kai K, Moriyama $M$, Narahara $\mathrm{H}$ : CCAAT/enhancer-binding protein a is epigenetically silenced by histone deacetylation in endometriosis and promotes the pathogenesis of endometriosis. J Clin Endocrinol Metab 2013, 98:E1474-E1482.

25. Nishida M, Nasu K, Fukuda J, Kawano Y, Narahara H, Miyakawa I: Down regulation of interleukin-1 receptor expression causes the dysregulated expression of CXC chemokines in endometriotic stromal cells: a possible mechanism for the altered immunological functions in endometriosis. J Clin Endocrinol Metab 2004, 89:5094-5100.

26. Nishida M, Nasu K, Ueda T, Fukuda J, Takai N, Miyakawa I: Endometriotic cells are resistant to interferon-gamma-induced cell growth inhibition and apoptosis: a possible mechanism involved in the pathogenesis of endometriosis. Mol Hum Reprod 2005, 11:29-34.

27. Miyagawa S, Maeda A, Takeishi S, Ueno T, Usui N, Matsumoto S, Okitsu T, Goto M, Nagashima $\mathrm{H}$ : A lectin array analysis for wild-type and 
a-Gal-knockout pig islets versus healthy human islets. Surg Today 2013, 43:1439-1447.

28. GlycoGene DataBase. [http://jcggdb.jp/rcmg/ggdb/]

29. Cummings RD: The repertoire of glycan determinants in the human glycome. Mol BioSyst 2009, 5:1087-1104

30. Arnaud J, Audfray A, Imberty A: Binding sugars: from natural lectins to synthetic receptors and engineered neolectins. Chem Soc Rev 2013, 42:4798-4813.

31. Seeberger PH, Werz DB: Synthesis and medical applications of oligosaccharides. Nature 2007, 446:1046-1051.

32. Turnbull JE, Field RA: Emerging glycomics technologies. Nat Chem Biol 2007, 3:74-77.

33. Song $X$, Lasanajak Y, Xia B, Heimburg-Molinaro J, Rhea JM, Ju H, Zhao C, Molinaro RJ, Cummings RD, Smith DF: Shotgun glycomics: a microarray strategy for functional glycomics. Nat Methods 2011, 8:85-90.

34. Dennis J, Waller C, Timpl R, Schirrmacher V: Surface sialic acid reduces attachment of metastatic tumour cells to collagen type IV and fibronectin. Nature 1982, 300:274-276.

35. Dennis JW, Laferte S, Waghorne C, Breitman ML, Kerbel RS: Beta1-6 branching of Asn-linked oligosaccharides is directly associated with metastasis. Science 1987, 236:582-585.

36. Taniguchi N, Miyoshi E, Ko JH, Ikeda Y, Ihara Y: Implication of Nacetylglucosaminyltransferases III and V in cancer: gene regulation and signaling mechanism. Biochim Biophys Acta 1999, 1455:287-300.

37. Brockhausen I: Pathways of O-glycan biosynthesis in cancer cells. Biochim Biophys Acta 1999, 1473:67-95.

38. Sorensen AL, Reis CA, Tarp MA, Mandel U, Ramachandran K Sankaranarayanan V, Schwientek T, Graham R, Taylor-Papadimitriou J, Hollingsworth MA, Burchell J, Clausen H: Chemoenzymatically synthesized multimetric Tn/STn MUC1 glycopeptides elicit cancer-specific antig-MUC1 antibody responses and override tolerance. Glycobiology 2006, 16:96-107.

39. Napoletano C, Rughetti A, Tarp MPA, Coleman J, Bennett EP, Picco G, Sale P, Denda-Nagai K, Irimura T, Mandel U, Clausen H, Frati L, Taylor-Papadimitriou J, Burchell J, Nuti M: Tumor-associated Tn-MUC1 glycoform is internalized through the macrophage galactose-type C-type lectin and delivered to the HLA class I and II compartments in dendritic cells. Cancer Res 2007, 67:8358-8367.

40. Sato T, Furukawa K, Greenwalt DE, Kobata A: Most bovine milk fat globule membrane glycoproteins contain asparagine-linked sugar chains with GalNAc beta 1 $\rightarrow$ 4GIcNAc groups. J Bioehem 1993, 114:890-900.

41. Stenman UH, Leinonen J, Alfthan H, Rannikko S, Tuhkanen K, Alfthan O: A complex between prostate-specific antigen and alpha-antichymotrypsin is the major form of prostate-specific antigen in serum of patients with prostate cancer: assay of the complex improves clinical sensitivity for cancer. Cancer Res 1991, 51:222-226.

42. Benchimol SS, Fuks A, Jothy S, Beauchemin N, Shirota K, Stanners CP: Carcinoembryonic antigen, a human tumor marker, functions as an intercellular adhesion molecule. Cell 1989, 57:327-334.

43. Wojciechowicz DC, Park PY, Datta RV, Paty PB: CEA is the major PHA-Lreactive glycoprotein in colon carcinoma cell lines and tumors: relationship between K-ras activation and beta1-6 branching of N-linked carbohydrate on CEA. Biochem Biophys Res Commun 2000, 273:147-153.

44. Magnani JL, Nilsson B, Brockhaus M, Zopf D, Steplewski Z, Koprowski H, Ginsburg VA: A monoclonal antibody-defined antigen with gastrointestinal cancer is a ganglioside containing sialylated lacto- $N$-fucopentaose II. J Biol Chem 1982, 257:14365-14369.

45. Berkes E, Mužinić A, Rigo J Jr, Tinneberg HR, Oehmke F: The analysis of the human plasma N-glycome in endometriosis patients. Eur J Obstet Gynecol Reprod Biol 2013, 171:107-115.

46. Kitamura N, Guo S, Sato T, Hiraizumi S, Taka J, Ikekita M, Sawada S, Fujisawa $\mathrm{H}$, Furukawa K: Prognostic significance of reduced expression of beta$\mathrm{N}$-acetylgalactosaminylated $\mathrm{N}$-linked oligosaccharides in human breast cancer. Int J Cancer 2003, 105:533-541.

47. McMahon RF, McWilliam LJ, Mosley S: Evaluation of three techniques for differential diagnosis of prostatic needle biopsy specimens. J Clin Pathol 1992, 45:1094-1098

48. Brauer $\mathrm{K}$, Hartig W, Bigl V, Bruckner G: Distribution of parvalbumin-containing neurons and lectin-binding perineuronal nets in the rat basal forebrain. Brain Res 1993, 631:167-170.
49. Sakiyama T, Kabayama M, Tomita M, Nakamura J, Mukai H, Tomita $Y$, Furukawa K: Distribution of glycoproteins with beta- $N$ acetylgalactosaminylated $\mathrm{N}$-linked sugar chains among bovine tissues. Biochim Biophys Acta 1998, 1380:268-274.

50. Buckhaults $P$, Chen L, Fregien N, Pierce M: Transcriptional regulation of $\mathrm{N}$-acetylglucosaminyltransferase V by the src oncogene. J Biol Chem 1997, 272:19575-19581.

51. Takahashi T, Ikeda Y, Miyoshi E, Yaginuma Y, Ishikawa M, Taniguchi N: Alpha1,6 fucosyltransferase is highly and specifically expressed in human ovarian serous adenocarcinomas. Int J Cancer 2000, 88:914-919.

52. Huang J, Liang JT, Huang HC, Shen TL, Chen HY, Lin NY, Che MI, Lin WC, Huang MC: Beta1,4-N-acetylgalactosaminyltransferase III enhances malignant phenotypes of colon cancer cells. Mol Cancer Res 2007, 5:543-552.

53. Hsu WM, Che MI, Liao YF, Chang HH, Chen CH, Huang YM, Jeng YM, Huang J. Quon MJ, Lee H, Huang HC, Huang MC: B4GALNT3 expression predicts a favorable prognosis and suppresses cell migration and invasion via $\beta_{1}$ integrin signaling in neuroblastoma. Am J Pathol 2011, 179:1394-1404.

doi:10.1186/1477-7827-12-100

Cite this article as: Hirakawa et al.: Wisteria floribunda agglutinin-binding glycan expression is decreased in endometriomata. Reproductive Biology and Endocrinology 2014 12:100.

\section{Submit your next manuscript to BioMed Central and take full advantage of:}

- Convenient online submission

- Thorough peer review

- No space constraints or color figure charges

- Immediate publication on acceptance

- Inclusion in PubMed, CAS, Scopus and Google Scholar

- Research which is freely available for redistribution 